\title{
REFLECTIVE SIMULATION FOR ON-LINE WORKLOAD PLANNING AND CONTROL
}

\author{
Roberto Revetria \\ Advanced Manufacturing Technology Group \\ University of Genoa, Savona Campus \\ Via Cadorna, 2 \\ Savona, SV 17100, ITALY
}

\begin{abstract}
Since its beginning, simulation has been used to study complex systems in order to infer on their future behavior, in this field several applications have been made using it as off-line tool for strategic level choices. In modern application, especially in the field of industrial automation, on-line simulation has been extensively used for supporting operative decision trough a classical schedulesimulate loop. The paper presents an application of on-line simulation to the distribution logistics sector: a department store is here controlled by on-line simulators able to help decision maker to decide how many counters to kept opened or how many people to use for shelves replenishment. Since this exercise could seriously affect the performances of a real life department store, the methodology is, in fact, very sensible to parameter settings, a nested simulator has been implemented and used for algorithm fine tuning and critical parameter choice.
\end{abstract}

\section{INTRODUCTION}

Simulation has proven to be very effective in supporting schedule evaluation for complex systems in several industrial applications. In distribution logistics industry there is a clear relation among picking reduction time and profitability. The more the picking and kitting times are reduced the more orders can be accomplished in one shift, resulting in an increase of the profitability rate. In the past, the staff scheduling required for assigned picking tasks to operators was executed at the beginning of the shift while today a continuous rescheduling activity has to be made in order to increase the response readiness to meet the replenishment take times.

The problem is not even a matter of scheduling performance, since a schedule will never be respected, it is more a matter of robustness of the planning. In such scenario simulation can play a crucial role since it offers the possibility of quantitatively evaluate a planning performance when the original planning scenario has

\author{
Flavio Tonelli
}

DIPTEM, Dipartimento di Ingegneria della Produzione, Termoenergetica e Modelli Matematici

Via all'Opera Pia, 15

Genova, GE 16145, ITALY

changed. This simulation-in-the-loop decision making process can be effectively evaluated by simulating a decision support system that has simulation in its internal logic: this is the case of the proposed reflective simulation model. The paper proposes an application of reflective simulation able to evaluate a on-line simulation-in-the-loop scheduling system.

\section{QUASI BLACK BOX MODELING}

There is a wide class of phenomena involving intelligent agents (i.e. customer behavior in a shop, drivers at a toll plaza) that can be accurately modeled only by having an exact knowledge of the internal agent's behavior. Such knowledge can be obtained at the price of long data collection campaign, multiple agent tracing, social behavior modeling and many other time-consuming techniques.

In the supermarket distribution industry, there is a growing interest in the possibility of accurately modeling the customer behavior in order to optimize the number of tellers to be opened in each period or the best way to use workers for shelves replenishment. In a supermarket, in fact, a stock out event is generally associated with a potential lost sale whose criticality is proportional to the duration of the stock out and the number of the items involved.

Another important issue is related to the average time spent by a single customer into the shop, it is not a secret that long waiting time in the teller queue seriously affects the profitability of the shop. This point is becoming more important in some countries, like Italy, where a significant portion of the fresh product is sold by operators inside a special area of the supermarket. In Italy, in fact, customer perception of the quality of the same raw ham pre-packed in self-service or sliced-at-the-moment could be dramatically different, resulting in a consistent sale loss at the short term and a consequent customer loss in the medium term for a only self service supermarket. In the 


\section{Revetria and Tonelli}

recent years, several techniques have been used to trace customer behavior into shops ranging from RFID tag on the trolley up to dedicated scanning camera placed at selected points of the shops. The results were generally poor, since the natural variability of the process drives very quickly the observation out of control.

Another important issue is related to external driven variability: in bad weather days the number of customer spending their off-duty afternoon in supermarket or department store increase dramatically, again there is a great interest in supermarket industry in forecasting queue length and customers' waiting times according to the weather change.

Since many agent-based simulation projects failed in the past for the lack of credible data in the customer behavior modeling, a different approach should be used.

In the proposed approach we abandoned the agent based behavior-modeling chimera in favor of a quasi-black box approach where some parameters can be actively measured and used for an input/output model.

In real life application a supermarket can be summarized as black box where customers enter at the entrance gate, spend some time in purchasing - according to their internal needs - and present themselves to the tellers for paying.

While it is quite simple to measure the input rate, it is very hard to investigate the single entity choice. The other important measures that can be conducted on the supermarket are: the post teller output rate and the check composition. While the first is only a mediated measure of the teller efficiency, the second could be used for obtaining an impressive list of information such as estimation of the average time spent in purchasing and/or in internal queues.

At this point a quasi-black box simulator can be used during regular operating hours to investigate the present system for possible evolution and to choose the best performing tactics. Because of the continuous updating of the simulator, an evolving scenario can be easily accommodated.

\section{REFLECTIVE SIMULATION USING SIMULA}

The use of on-line simulation for supporting complex decision making in evolving scenario has been widely documented in several industrial applications; however the great part of those was mainly related to manufacturing industry where the extensive use of automation transformed the data collection into a matter of software integration. In supermarket industry, it is simply impossible to track customer behavior without high cost and without violating the privacy protection act.

It is not possible to study the customer purchasing behavior at entity level so it is very difficult to implement an agent based simulation to support the teller opening schedule. On the other side the use of the quasi-black box simulation methodology requires a credible validation in order to be fine-tuned and adopted into a real life application. The need of a test bed for the quasi-black box methodology requires the adoption of a model able to reproduce an internal simulation and its decision making process while simulating normal operation procedure and scenario evolution.

The authors addressed this issue to the use of the nested simulation where the outer model will be used to simulate the real world and the nested simulation will be used to act as the on-line simulator and its consequent Decision Making Process. In the proposed application a simple supermarket model is modeled in the outer simulator, the customer arriving process is modeled by using a uniform distribution of the mean time between arrivals. A multiple period simulation exercise is used to simulate up to 9 different customer arriving rates during a single day. In this way a possible perturbation path can be actively modeled by continuously adjusting the arriving rate.

In the same simulator a nested simulation is implemented using the same logic but obtaining its distribution data from statistics of the outer simulator. Practically the inner simulator can obtain the customer arriving ratio and the customer mean processing time from sample made in the "Real World". Like in reality, the simulator can only investigate the possible evolution of the "Real World" from the incomplete samples "visible" in different points.

In other words only the Arriving Process and the Leaving Supermarket process are visible and all the other information can only be guessed, particularly the customers' Inter Arrival Time (IAT) statistics is collected by a sensor placed near the entrance door. In this way the inner simulation has the visibility on the real world similar to the one that it will have in the real application.

During the simulation of the "Real World", the user will be prompted for a choice made on the possible scenario evolution computed from the present point and according to a predefined teller opening scheme. This fact leads to multiple time axes departing from the "Real" to the various "Virtual" worlds. Since internal statistics are continuously updated the inner simulation can be used to investigate the possible reaction to an evolving scenario.

The nested simulation model can now be applied to the quasi-black box methodology to test the effectiveness of the approach.

Among the various tools and languages suitable for simulation application, the authors decide to implement the technological demonstrator using SIMULA, a generalpurpose language with a specific capability for nested simulation. This choice was driven following the literature that indicate in SIMULA and in Java (Kindler et al. 1997) the two languages capable of supporting complex exercises in nested simulation. 


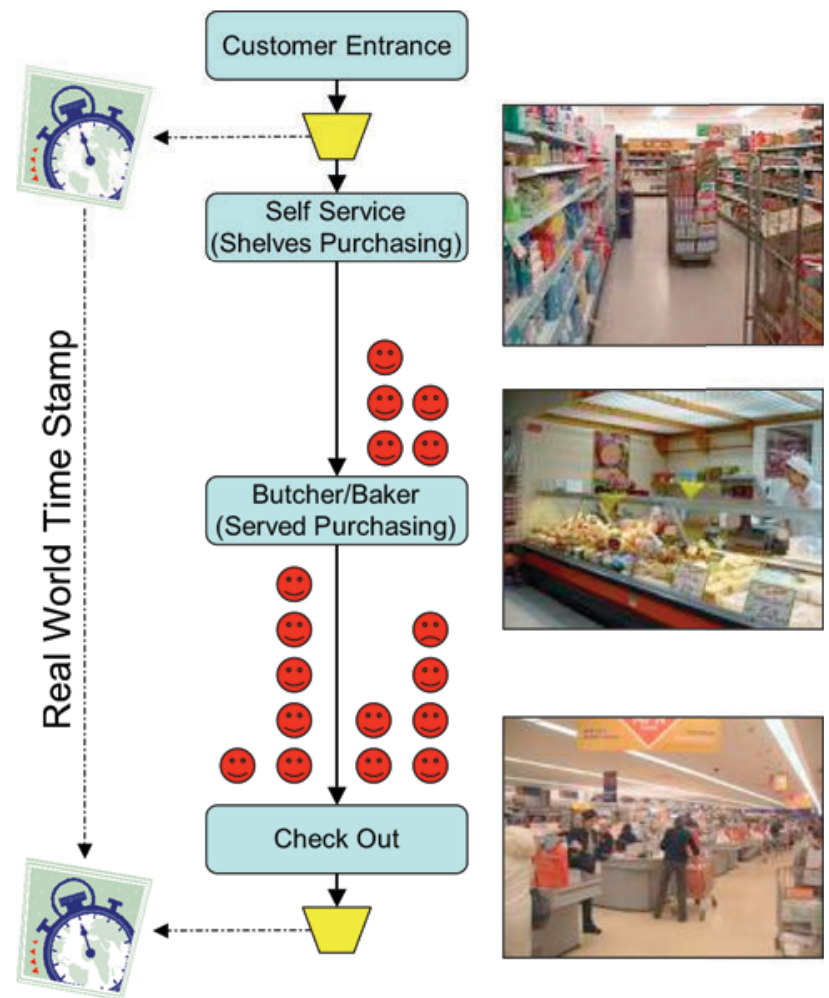

Figure 1: General application scheme

Life rules of Simula objects are coroutines, which may be temporarily stopped and later resumed. There are two levels of (quasi)parallelism in Simula. First level does not work with time, the programmer "thinks in quasiparallel". The second level introduces the notion of time, the programmer works with parallel processes. First level can be applied to all classes, the second level is implemented by the class Process of the system class Simulation. A quasiparallel system (QPS) in Simula is basically a block whose body creates some objects that together with the main block made up the system of quasiparallel coroutines. Because Simula (unlike for example Pascal) is a true block oriented language, a block (that generally means a QPS) can occur at any place in the code as a statement. It means that a QPS can contain local (nested) QPS's that can also contain local QPS's leading a complex structure of nested quasiparallel process. SIMULA is today available and supported in several commercial implementations; however the authors decided to use GNU Cim version 3.36. GNU Cim is a compiler for the programming language SIMULA (except unspecified parameters to formal or virtual procedures). It offers a class concept, separate compilation with full type checking, interface to external $\mathrm{C}$ routines, an application package for process simulation and a coroutine concept.

GNU Cim is a SIMULA compiler whose portability is based on the $\mathrm{C}$ programming language. The compiler and the run-time system is written in $\mathrm{C}$, and the compiler produces $\mathrm{C}$ code, that is passed to a $\mathrm{C}$ compiler for further processing towards machine code.

GNU Cim is copyrighted by Sverre Hvammen Johansen, Stein Krogdahl, and Terje Mjs, Department of Informatics, University of Oslo. Implemented simulation model is presented in Figure 2, where it is possible to see the customer enter into store and is "seen" by the entrance sensor providing a simple statistics for the Inner Customer Generator Model (Sklenar 1997).

The customer now has the chance to spend some time purchasing in the store in the self-service area (free service shelves) and, eventually, purchase some butchery or bakery products at the serviced desk. Butcher and bakery area are served by 12 clerks that maintain a separate queue for their services. An average $40 \%$ of the customers will require products from the butcher or bakery resulting in a delay in their purchasing process.

At the end of the purchasing process the customer will report himself to the counter for the check out, as first choice the shortest teller queue will be chosen. Since the time spent in queue is, usually, a critical factor in store management, a simple algorithm will implement the behavior of queue switching usually followed by the Italian customer.

The customer while in queue for a teller will "look around" in the adjacent queue investigating for a shorter queue. In case of an adjacent queue 2 people shorter than the present he entered he will jump to the end in order to save time. Jumping is not allowed into a closing queue.

If a queue is approaching closing, no more customers will be accepted in entrance and the counter will terminate its duties after the last customer in queue. An opening teller will, generally, cause queue switching in its first minutes of operating time.

The number of active tellers is defined at the beginning of the 12 simulation periods; customers eventually left in the shop at the end of a simulation will be served before closing. The outer simulator collects statistics on the arriving customers (minimum and maximum inter arrival time) and use it for the internal simulation.

At each simulated step the user was prompted for the opening teller choice, with the results of the simulation made in the "Virtual World" and immediately informed about the result of the simulation in the "Real World".

The use of SIMULA poses some difficulties in Verification \& Validation; such language, in fact, lacks the modern tool features for rapid GUI development, this is becoming very critical when compared to Arena $^{\mathrm{TM}}$, Simul8 ${ }^{\mathrm{TM}}$, ProModel ${ }^{\mathrm{TM}}$ tools that have nice tool for supporting simulation visualization.

In order to avoid this problem, authors used Wolverine Proof Animation ${ }^{\mathrm{TM}}$ that supports very high-fidelity visualization for general purpose simulation language. Proof Animation ${ }^{\mathrm{TM}}$ provides both off-line and on-line 
animation on Windows platforms and can be used by a wide variety of programs.

The implemented model introduced an internal Fresh Product shop inside the supermarket area, now customers have the chance to buy some fresh products directly in the Department Store and are served by a 4-server position that generates an internal queue system.

Again, the internal simulation, representing the "Virtual World", is driven by statistics generated by the outer simulation model, representing the "Real World". Control points of this new model are the customer arrival, obtained at the customer's entrance metering system, and the total percentage of customers spending their time into the Fresh Product Store (Butchery/Bakery) obtained with the customer check analysis.

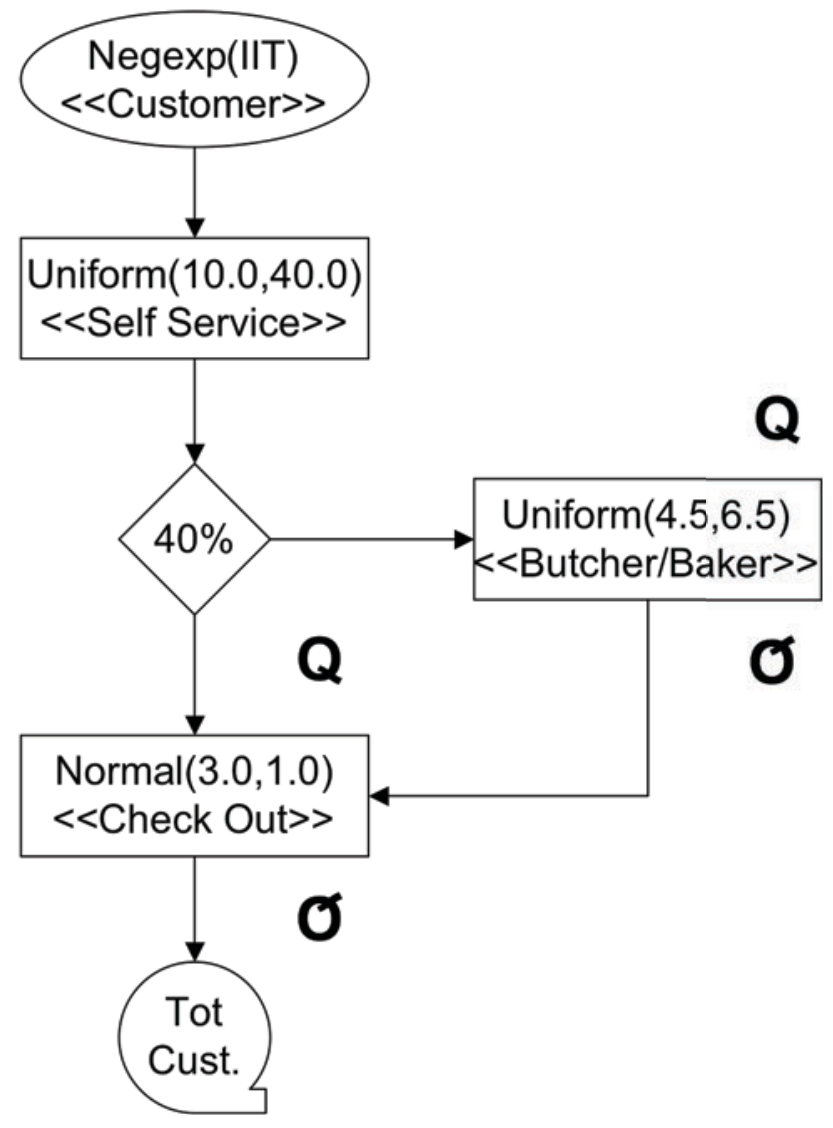

Figure 2: Implemented simulation model

\section{AN APPLICATION TO A REAL CASE}

Proposed application was tested with a complete implementation including Fresh Product internal store, 15 tellers row and a queue-balancing algorithm.

The simulator provides a 12 hour period covering the typical opening hours of an Italian Supermarket (8AM8PM), typical peak hour occurs at $10 \mathrm{AM}$ and 5 PM. Since the model was created to work also with a more complex time path (in order to be used later on another research) the generation of the load curve was embedded into the outer model itself using the following equation.

$$
J=10 J\left\{\left\{\frac{1}{2 \sqrt{2 J}} e^{j \frac{k+7 J p_{1}}{2 \sqrt{2}}}+\frac{1}{2 \sqrt{2 J}} e^{j \frac{k+7 J p_{2}}{2 \sqrt{2}}}\right\} J j\left\{1.5+\frac{1}{19} J(19 \mathrm{~J} k+1)\right\}\right\}
$$

where:

- $\lambda$ : is the customer arrival rate, measured in customers' arrivals per minute;

- $\quad \mathrm{p}_{1}$ : is morning peak time;

- $\mathrm{p}_{2}$ : is afternoon peak time;

This particular behavior (see Figure 3) is able to introduce a level of complexity into the model. Since the internal simulation model is driven by external model statistics a 1 hour delay between increasing or decreasing IAT and internal model awareness is introduced as possible oscillating path (Banks et al. 2005), this point has been discussed in (Bruzzone and Revetria 1999) and can be properly addressed using Artificial Neural networks as Computing Anticipatory Model for the IAT time path (Bruzzone et al. 2001).

At the same time the implemented model can be used to investigate the benefit of innovative methodologies for products replenishments over the shelves as discussed in (Bruzzone, Revetria, Genovese and Rombi 2001).

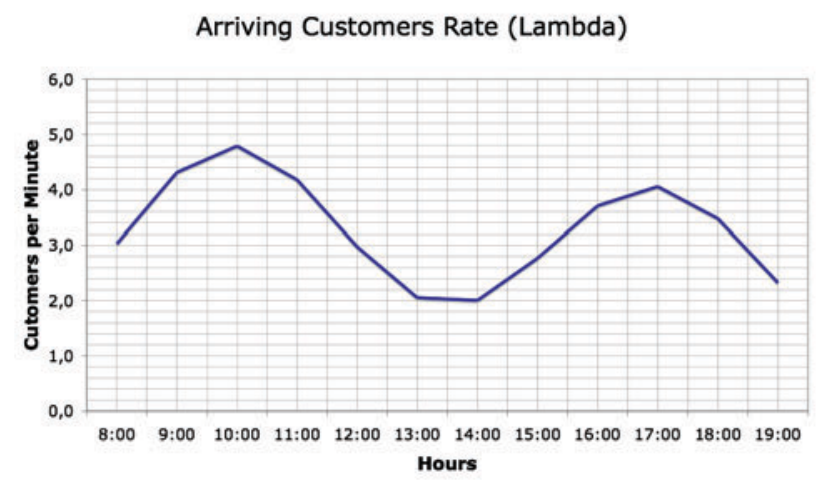

Figure 3: Workload implemented

Implemented model requires only a data collection for the teller process and the internal Fresh Product store, both internal (nested) and external (outer) simulation models works on the same statistics implementing a Standard Random Generator for the teller process and a Uniform Random Generator for the internal Fresh Product Store.

The internal simulation serves all the possible configurations from 1 to 15 opened tellers driving the possibility to investigate how many tellers keep opened in each of the 12 hours of the day shift.

Since there is no knowledge about the number of customer in queue at the end of each "Real World" hour, the internal simulator may start with a warm up period of 15 minutes. Investigations made with a complete vision of the queue from the internal simulation demonstrate that the 


\section{Revetria and Tonelli}

effectiveness of this choice was widely proven. For a complete discussion of this point and a quantitative evaluation of the Mean Squared Pure Error see (Revetria et al. 2006).

For the experimentation 2 models were implemented, the first has a negative exponential IAT distribution in the outer model and a uniform distribution in the inner model, this choice was able to demonstrate the applicability of the proposed methodology to a "maximum ignorance case"; the second model implements correctly a negative exponential distribution. In both cases the parameters of the distribution were extracted from statistics made on the outer simulation model as in a real life scenario where data collection is carried out on the real system. The first experimental results present (Figure 4) a poor performance; the systems appear to be ineffective in controlling TIS output variable resulting in an increase of the queues and in a reduction of the total customers served.

The simulation results for the second scenario are presented in Figure 5, showing a good capability of the system in controlling the TIS output variable also in the presence of an oscillating input behavior.

Uniform Model Divergent Behavior

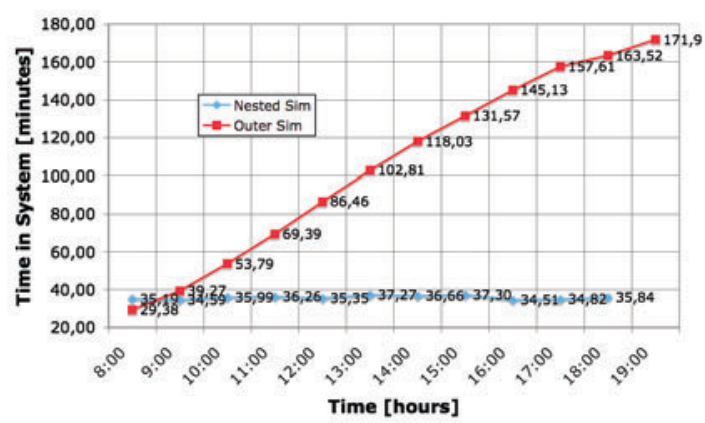

Figure 4: Poor performances of uniform model

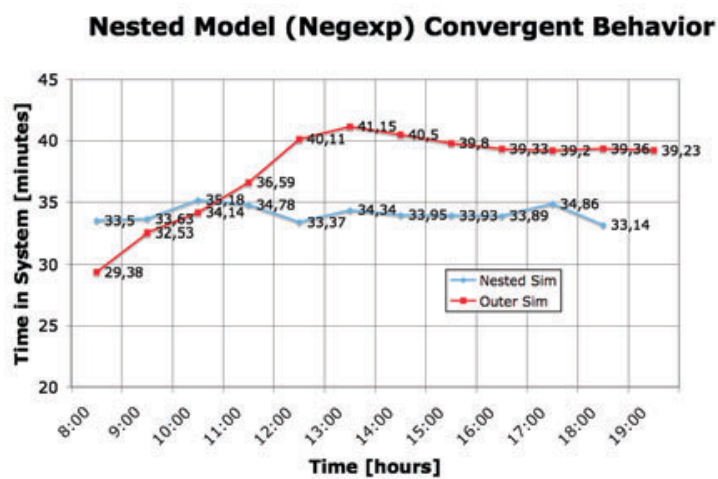

Figure 5: Controlling capability of the implemented nested model

The proposed methodology presents some benefits also in term of increasing the overall productivity of the shops; the first experiment, in fact, ended with only 1411 customer served in a 12 hours opening time frame against the 2217 obtained in the second experiment with a net increment of $57 \%$.

\section{CONCLUSIONS}

The use of on-line simulation for controlling queue parameters and increase performances of a department store has proven to be very effective also in case of application of a quasi-black box modeling paradigm.

Benefits can be studied well before implementation by using a nested simulation approach that is proper for this application domain. Among various simulation software SIMULA $^{\mathrm{TM}}$, a 50-year-old language based on ALGOL ${ }^{\mathrm{TM}}$, is still in shape and is the right choice for a very effective implementation.

A practical application of the proposed methodology has been presented and discussed.

\section{REFERENCES}

Banks, J., J. S. Carson, B. L. Nelson, and D. M. Nicol. 2005. Discrete-event system simulation. 4th ed. Upper Saddle River, New Jersey: Prentice-Hall, Inc.

Bruzzone A., R. Mosca, A. Orsoni, and R. Revetria. 2001 Forecasts modelling in industrial applications based on ai techniques. International Journal of Computing Anticipatory Systems (extended from Proceedings of CASYS2001, Liege Belgium August 13-18) 11:245258, ISSN1373-5411

Bruzzone A.G. and R. Revetria. 1999. Artificial neural networks as support for logistics in super-market chains. In Proceedings of HMS99, Genoa, September 16-18

Bruzzone A., R. Revetria, M. Genovese, and L. Rombi. 2001. Inventory management by integrating ERP systems and simulation tools. In Proceedings of SCI2001, Orlando, July 22-25

Blümel, P. and E. Kindler. 1997. Simulation of antagonist mutually simulating systems - first experiences. SimVis 1997: 56-65

Revetria R. 2001. Replenishment policy optimization using the simulation and the fuzzy logic approach. In Proceedings of The SCI2001 Conference, Orlando FL, July 22-25

Revetria R., C. Forgia, and Catania A. 2006. Agent based vs nested simulation for supporting on-line teller scheduling in groceries supermarket distribution: a case study. In Proceedings of ESM2006, LAAS, Toulouse, France October 23-25, 2006

Sklenar J. 1997. Introduction to SIMULA, available on <http://staff.um.edu.mt/jskl1/talk.h tml $>$ [accessed April 2007] 


\section{AUTHOR BIOGRAPHIES}

ROBERTO REVETRIA is Associate Professor in Mechanical Engineering and Logistics in the University of Genoa. He is consultant in several projects involving simulation with special attention to Distributed Discrete Event and Agent Based Continuous Simulation. He is teaching Modeling \& Simulation, VV\&A, Distributed Simulation (HLA), Project management in Master Courses Worldwide and he is teaching Industrial Plants Design, Maintenance Management in University of Genoa Masters' Courses. He is member of SCS, IASTED, ACM, ANIMP, AICE, MIMOS and LSC.

FLAVIO TONELLI is a researcher in Mechanical Engineering and Operation management. He has his degree in Electronic Engineering and his $\mathrm{PhD}$ in Management Engineering from Genoa University. He is expert in using Modsim III and developer Osiris/WILDNESS Simulation package. He is a major Expert in Operation Management, MSDD and Lean Management Methodology. $\mathrm{He}$ is currently an accredited teacher in several International Master Courses. He successfully led several research projects (European Union cooperation with Czech Republic partners). Currently he is charge of development of Scheduling, Planning and Execution System in cooperation with Atomos SpA. 